\title{
El examen del Icfes -Saber 11-y la prueba de entrada como predictores del aprendizaje de los estudiantes de primer semestre de psicología de una universidad privada de Bogotá ${ }^{1}$
}

\author{
CARLOS ANTONIO PARDO ADAMES ${ }^{2}$, KAREN CABARCAS ACOSTA ${ }^{3}$ \\ UNIVERSIDAD CATÓLICA DE COLOMBIA
}

Recibido, diciembre 01 de 2014

Concepto evaluación, febrero 24 de 2015

Aceptado, marzo 26 de 2015

\begin{abstract}
Referencia: Pardo Adames, C.A.; Cabarcas Acosta, K. (2015). "El examen del Icfes -Saber 11- y la prueba de entrada como predictores del aprendizaje de los estudiantes de primer semestre de Psicología de una universidad privada de Bogotá". Revista Academia y Virtualidad, 8, (1), 88-98
\end{abstract}

\section{Resumen}

Es una tradición en Colombia que las Instituciones de Educación Superior utilicen diversos procesos para el ingreso de los estudiantes a sus programas y el de mayor uso es el Examen de Estado Saber 11, requisito obligatorio como elemento de admisión. Igualmente, éstas utilizan pruebas diseñadas por las instituciones como un elemento adicional de ingreso. Este estudio plantea la pregunta de si estas pruebas en realidad constituyen buen material para incluirlas como parte determinante del ingreso de estudiantes o si su utilidad es restringida y es mejor utilizar otros mecanismos complementarios. Los resultados indican que ambas pruebas se relacionan en forma significativa con el resultado del aprendizaje de los estudiantes medido a través de las notas obtenidas durante el primer semestre, aunque la prueba Saber 11 tiene una relación negativa. El porcentaje de varianza explicado por las pruebas de entrada es $27 \%$, lo que implica que sería pertinente ampliar el alcance del estudio e incluir otras variables potencialmente relacionadas.

Palabras clave: educación, calidad de la educación, aprendizaje.

\section{The ICFEs Test -Saber 11- and Admission Test as learning predictors of Psychology first semester students}

\begin{abstract}
1. Artículo de Investigación Científica y Tecnológica.

2. Docente y Director de Docencia Facultad Psicología. Contacto: capardo@ucatolica.edu.co

3. Docente. Contacto: klcabarcas@ucatolica.edu.co
\end{abstract}

Traditionally Colombian higher education institutions have different processes for admission of students to their programs and most of them apply the relevant, required State Test Saber 11. They also use tests designed by themselves as an extra item to admission. This study asks the question whether these tests are actually good stuff to include them as a crucial part of the admission of students, but otherwise usefulness is restricted and it is better to use other supplementary mechanisms. Results show both tests are significantly related to the learning result by students 
measured through grades got during the first half, although the Test Saber 11 gives a negative ratio. The variance percentage explained by admission test is 27 percent, involving to extend the scope of the study to include other potentially related variables.

Keywords: education, quality of education, learning.

\section{O exame ICFES-Saber 11 e a prova de ingresso como predições do aprendizado dos estudantes de primeiro semestre de psicologia de uma universidade privada de Bogotá}

\section{Resumo}

Já é uma tradição na Colômbia que as Instituições de Educação Superior empreguem diversos processos para o ingresso dos estudantes a seus programas e o mais usado é o Exame de Estado Saber 11, requisito obrigado para a admissão. Igualmente, as instituições de Educação Superior empregam provas desenhadas por elas mesmas como um requisito adicional para o ingresso. Este estudo propõe a pergunta se estas provas na realidade constituem um bom material para as incluir como elemento determinante para o ingresso dos estudantes ou se a sua utilidade é restringida e seria melhor empregar outros mecanismos complementários. Os resultados indicam que ambas provas relacionamse significativamente com o resultado do aprendizado dos estudantes medido através das notas obtidas durante o primeiro semestre, embora a prova Saber 11 tenha uma relação negativa. A porcentagem de variância explicado pelas provas de entrada é de $27 \%$, o que implica que seria pertinente ampliar o alcance do estudo e incluir outras variáveis potencialmente relacionadas

Palavras-chave: educação, qualidade da educação, aprendizado.

\section{Introducción}

En cada ocasión que se publican resultados de evaluaciones en educación se desarrolla un gran debate sobre la calidad de la educación, no sólo en nuestro país sino en la mayoría de los países participantes en esas evaluaciones. Quejas de unos con respecto de los otros, exigencias de parte y parte como factor fundamental para mejorar y un gran clamor de la sociedad por educar bien a los futuros ciudadanos.

En 1968 se crea en Colombia el Instituto Colombiano para el Fomento de la Educación Superior (ICFES), que tiene dentro de sus dependencias al Servicio Nacional de Pruebas (SNP), el cual se desarrolla a partir de la reestructuración que tiene el Servicio de Admisión Universitaria y Orientación Profesional, que hasta esa fecha se encargaba, dentro de múltiples funciones, de la elaboración, administración y evaluación de instrumentos que fueran útiles a las instituciones de Educación Superior para la selección de los estudiantes que ingresarían a sus aulas; sin embargo, la aplicación de las mismas se hacía, siempre y cuando, la institución universitaria así lo solicitara. Para el año de creación del ICFES, el SNP lleva a cabo la primera aplicación masiva de una prueba de selección que se conoció con el nombre de Exámenes Nacionales y estuvo conformada por cuatro (4) pruebas de aptitudes (matemática, verbal, razonamiento abstracto, relaciones espaciales) y cinco pruebas de conocimiento (ciencias sociales y filosofía, química, física, biología e inglés) (Grupo de Evaluación de la Educación Básica y Media, 1999). 
El examen del Icfes -Saber 11-y la prueba de entrada como predictores del aprendizaje de los estudiantes de primer semestre de psicología de una universidad privada de Bogotá

Es solo hasta 1980, con el decreto 2343 (Ministerio de Educación Nacional, 1980), que se reglamentan los Exámenes de Estado para el Ingreso a la Educación Superior y se establece la obligatoriedad de los mismos, el decreto establece que estos tienen como objetivo comprobar la existencia de niveles mínimos en las aptitudes y áreas de conocimiento antes mencionadas en aquellos individuos que aspiren a ingresar a las instituciones que conforman lo que se dio a llamar como el sistema de Educación Superior.

El decreto también establece que la aplicación del examen les permitirá a las instituciones de Educación Superior tener un punto de referencia que les facilite la toma de decisiones frente a la selección y admisión de sus estudiantes, quienes además contarían con una evaluación considerada como homogénea.

El Ministerio de Educación (1980) a través del decreto 2343, también estableció que las instituciones de Educación Superior deben exigir un puntaje mínimo obtenido en el examen de estado para el ingreso de sus estudiantes, aunque les permitía ponderar este resultado de acuerdo con las características del programa al que se aspiraba el ingreso.

El Ministerio de Educación Nacional (1992), a través de la Ley 30 ("por la cual se organiza el servicio público de la Educación Superior"), ratifica la obligatoriedad del examen de Estado y lo establece como un requisito para el ingreso a la Educación Superior, poniéndolo al mismo nivel del título de bachiller.

Entre los años 1980 y 1990, el examen de estado estuvo conformado por 9 pruebas, que se reorganizaban en 5 áreas, a saber ciencias naturales, lenguaje, matemáticas, ciencias sociales, y una prueba electiva. El examen arrojaba tres tipos de puntajes, el primero hacía alusión a cada una de las pruebas e iba de 20-80 puntos; el segundo era un puntaje por área que era el resultado del promedio de las pruebas que conformaban cada área; el tercero era un puntaje total que surgía del promedio de las cinco áreas (Grupo de Evaluación de la Educación Básica y Media, 1999).
A partir de la mitad de la década de los 90s, el examen de estado sufre modificaciones que surgen, especialmente, de la renovación de los propósitos educativos (Ley 115 de 1994), de los resultados de las investigaciones internas desarrolladas por el ICFES y de la necesidad de ajustar el instrumento a las nuevas condiciones de la evaluación de la calidad de la Educación Superior (Grupo de Evaluación de la Educación Básica y Media, 1999).

Ya desde la formulación de los lineamientos curriculares (Ministerio de Educación Nacional, 1998) se inicia un cambio en la formulación de políticas de educación, en las que se pretende virar hacia una educación por competencias, habilidades, aptitudes y valores y no solo por conocimientos. A partir de la publicación de los estándares de evaluación, queda claro que lo que le interesa al Estado es una formación por competencias, de tal manera que el ICFES, y específicamente el SNP no son ajenos a estas necesidades y se reformula la prueba de estado que pasa a llamarse Saber 11.

Las pruebas Saber y en especial la prueba Saber 11, busca hacer una evaluación por competencias, ya que se supone que el objetivo de la educación básica y de la educación media es el desarrollo de competencias genéricas o "competencias para la vida", necesarias para que el individuo tenga un adecuado desempeño social, laboral y cívico y que son independientes a la formación en una profesión u oficio particular. El Ministerio de Educación Nacional (MEN) propone además que ya que las competencias genéricas son básicas para el adecuado desempeño laborar, las instituciones de Educación Superior están llamadas a fortalecerlas a través de la elaboración y aplicación de sus currículos (BernalVelásquez, 2013).

En el año 2000 la prueba Saber 11 (conocida en la época como Prueba ICFEs), tiene un viraje que le permite hacer no solo una evaluación por competencias sino, además, utilizar la Teoría de Respuesta al Ítem (TRI) (Pardo, 1999) para analizar los resultados de los estudiantes que se sometían a esta, lo que contribuyó a que Colombia se ubicará a nivel internacional como uno de los países a la vanguardia de la evaluación por competencias. En realidad, 
el ICFEs ha utilizado la TRI desde 1991 en las evaluaciones conocidas como Saber o evaluación de la calidad de la educación en los grados 3 y 5 de educación básica (Pardo, 1998) siendo el primer país de Latinoamérica en hacerlo en los procesos de evaluación educativa. La Teoría de Respuesta al Ítem marcó una diferencia de fondo en relación con la forma como se trabajaba en el ICFES, por lo menos en los que a la prueba de Saber 11 hace referencia, ya que en este contexto se utilizaba la Teoría Clásica de los Test como elemento central del procesamiento de datos y diseño de evaluaciones. La estructura de la prueba Saber 11, se diseñó bajo el esquema de las áreas curriculares que se propusieron en los Lineamientos Curriculares y la estructura aún se mantiene. Saber 11 está compuesto por dos componentes (Bernal-Velásquez, 2013):

El primero corresponde a un núcleo común constituido por las siguientes pruebas que son aplicadas a todos los estudiantes que responden el examen: Lenguaje, matemáticas, biología, física, química, ciencias sociales, filosofía e inglés. El segundo corresponde al núcleo flexible, compuesto por cuatro pruebas de profundización (profundización en matemáticas, profundización en lenguaje, profundización en sociales y profundización en biología) y dos interdisciplinares (medio ambiente y violencia y sociedad), la idea es que cada estudiante puede escoger una de las pruebas de acuerdo con sus intereses y solo responderá a ella (Bernal-Velásquez, 2013).

Hasta el primer semestre del año 2014, los resultados de la Prueba Saber 11, se presentaban tanto en un nivel individual como institucional. A nivel individual los estudiantes recibían un reporte en el que se les informaba el puntaje que obtuvo en cada una de las pruebas del núcleo común, en una escala con promedio de 50 y desviación 10. Adicionalmente, se les informaba el puntaje y el nivel en el que se encontraban para cada una de los componentes y las competencias que conforman las pruebas y finalmente se le indicaba el puesto en el que se encontraban ubicado en relación con los demás estudiantes que respondieron la prueba. El ICFES propone que los resultados del examen Saber 11, sean utilizados entre otros usos como un requisito para el ingreso a la Educación Superior, especialmente si se considera que el examen busca determinar en qué nivel los estudiantes cuentan con las competencias genéricas requeridas para un adecuado desempeño laboral posterior. Igualmente, establece que algunas instituciones de Educación Superior utilizan los resultados del Saber 11 como información básica para generar cursos de nivelación que favorezcan la permanencia de los estudiantes en el programa académico (Bernal-Velásquez, 2013).

Para el segundo semestre del año 2014, se llevó a cabo la aplicación de un examen Saber 11, cuyos resultados son comparables con los arrojados por las evaluaciones que se aplican en los otros niveles de educación del país como Saber 3, Saber 5, Saber 9 y Saber Pro. En general, los cambios del nuevo Saber 11 implican la inclusión de la prueba sobre competencias ciudadanas que conforman la prueba de Sociales y ciudadanas; diferenciación en la prueba de matemáticas de lo que sería competencias genéricas y lo que no; y la fusión de pruebas que se estableció evaluaban competencias genéricas en común (filosofía y lenguaje que se pasó a llamar lectura crítica; física, química y Biología, se unieron y conforman lo que se conoce como ciencias naturales que además incluye ciencias, tecnología y sociedad). Por último se eliminó el componente flexible (Pedraza-Daza, Bernal-Velásquez \& Mora-Monje, 2014).

Los resultados que recibirá el estudiante estarían dados en los siguientes niveles: a) puntaje global que va de 0 a 500, con una media de 250 y una desviación de 50; b) puesto, están definidos 1000 puestos; c) puntaje por pruebas y subpruebas que va de 0 a 100 , con media de 50 y desviación de 10; d) nivel por pruebas y subpruebas, que serían $\mathrm{A}+, \mathrm{A}, \mathrm{B}$ y $\mathrm{C}$, para inglés la clasificación es A-, $\mathrm{A} 1, \mathrm{~A} 2, \mathrm{~B} 1 \mathrm{Y} \mathrm{B}+$; y e) decil por pruebas y subpruebas, que va de D01 (10\% inferior de la población) a D10 (10\% superior de la población) (Pedraza-Daza, BernalVelásquez \& Mora-Monje, 2014).

Aunque la institución en la que se realiza el estudio, como la mayoría de las instituciones de Educación Superior de Colombia, tiene dentro de sus requisitos de ingreso la presentación del examen, no hay una exigencia de un puntaje mínimo para ingresar. 
El examen del Icfes -Saber 11-y la prueba de entrada como predictores del aprendizaje de los estudiantes de primer semestre de psicología de una universidad privada de Bogotá

El proceso de selección que ha sido definido en la institución, se lleva a cabo por cada una de las facultades o programas. La facultad de Psicología ha establecido como requisito y procedimiento de selección de aspirantes lo siguientes pasos:

El estudiante debe anexar la certificación de presentación del examen de estado. Para estudiantes que hayan presentado el examen antes del año 2000 se exige un puntaje mínimo de 230, para quienes lo presentaron después de ese año no se exige un puntaje mínimo.

Luego se asigna una cita de entrevista la cual es realizada en la facultad por los docentes designados desde la Coordinación de Bienestar estudiantil. Estas entrevistas se hacen en formato grupal y evalúan cuatro aspectos: conocimiento general sobre la carrera; contextualización (aspectos por los cuales toma la decisión de estudiar psicología en términos personales y sociales; área familiar, aspecto que no tiene una calificación); y factores transversales e implícitos (expresión verbal, control de la ansiedad, manejo de la entrevista). Se da una puntuación total que va de 10 a 50 pts. Para que un estudiante sea aceptado debe obtener un puntaje entre 30 y 50; si obtiene un puntaje que se ubique en el rango entre 20 y 34 puntos el aspirante pasa a segunda entrevista, la cual es realizada por dos psicólogos clínicos. Puntajes inferiores a 19 puntos implican que no serán admitidos.

Una vez se realiza la entrevista los estudiantes deben responder la prueba de personalidad MINIMULT, la cual es tenida en cuenta como criterio de selección solo en los casos en los que los estudiantes pasan a segunda entrevista.

Cuando se han llevado a cabo todas las entrevistas, se envía un informe al área de admisiones informando los nombres de los aspirantes que fueron aceptados.

La Institución ha establecido que todos los estudiantes de primer semestre respondan una prueba de entrada que tiene como objetivos identificar su nivel de desarrollo y servir de insumo para la generación de estrategias que permitan el fortalecimiento del proceso educativo
(Forero, León \& Gutiérrez, 2014). La prueba evalúa 6 componentes estructurados desde las competencias genéricas, entendidas como "el conjunto de conocimientos, habilidades, actitudes, comprensiones y disposiciones cognitivas, metacognitivas, socio afectivas, comunicativas y psicomotoras apropiadamente relacionadas entre sí para facilitar el desempeño flexible, eficaz y con sentido de una actividad o de cierto tipo de tareas en contextos relativamente nuevos y retadores" (Vasco, 2003; citado por Bernal-Velásquez, 2013).

Los componentes de la prueba de entrada son: inglés, comprensión lectora, aptitud verbal, competencias ciudadanas, conocimientos matemáticos y razonamiento matemático. Con la prueba de inglés se pretende establecer si el estudiante cuenta con las competencias que le permitan la comprensión de la segunda lengua a través del manejo de estructuras básicas; con comprensión lectora se identifica la capacidad del individuo de leer compresivamente distintos textos a partir del uso de estrategias comunicativas y lingüísticas que permitan el análisis y la generación de relaciones entre los componentes del texto. Aptitud verbal se refiere a la habilidad con la que cuentan los estudiantes para usar las estrategias comunicativas y lingüísticas para el análisis y establecimiento de relaciones entre los componentes de un texto.

Competencias ciudadanas se refiere a las competencias para analizar el contexto dentro del marco ético propuesto desde la Constitución Política Colombiana. Con la prueba de conocimientos en matemáticas se busca establecer si el estudiante cuenta con los conocimientos básicos de geometría, aritmética y algebra; y por último la prueba de razonamiento matemático tienen como objetivo evaluar las competencias relacionadas con las habilidades que se requieren para la aplicación de los conceptos básicos de las matemáticas para la interpretación y análisis de datos (Forero, León \& Gutiérrez, 2014).

La prueba arroja resultados tanto cuantitativos como cualitativos. Se informa el nivel de desempeño por cada componente, pero también se brinda información relacionada con el porcentaje de ítems respondidos 
correctamente por cada estudiante en relación con el total de ítemes de cada componente (Forero, León \& Gutiérrez, 2014).

Los resultados de la prueba son procesados por el área de coordinación de evaluación de la Universidad y son enviados vía electrónica a los Decanos, Directores de Docencia y Coordinadores de evaluación de cada una de las Facultades, quienes deben tomar acciones pertinentes en relación con la generación de estrategias para el fortalecimiento de los estudiantes.

Es en ese marco en el que la Dirección de docencia y la coordinación de evaluación de la Facultad de Psicología proponen desarrollar un estudio que permita establecer si los resultados en el examen Saber 11 y los puntajes obtenidos en la prueba de entrada predicen el rendimiento académico de los estudiantes en aras de generar acciones que permitan garantizar o mejorar los niveles de permanencia de los estudiantes.

Para ello, además de la información anteriormente descrita sobre el Saber 11 y la prueba de entrada se cuenta con los resultados académicos de los estudiantes de primer semestre de 2014, los cuales son generados por los profesores que tienen a su cargo cada una de las asignaturas y que surgen de la conjunción de evaluaciones individuales y grupales que se desarrollan durante el semestre y que se distribuyen de la siguiente manera, un primer corte que corresponde al $30 \%$ de la nota total y que estaría evaluando los contenidos programáticos de las primeras cinco semanas de clases; un segundo corte que corresponde al $30 \%$ de la nota total y que comprendería la evaluación de los contenidos programáticos de las semanas 6 a la 10; y un último corte que corresponde al $40 \%$ de la nota total y que incluye la evaluación de los contenidos de la semana 11 a la 16. Algunos profesores consideran pertinente hacer una evaluación final acumulativa que pretende abarcar los contenidos vistos durante todo el semestre.

En este sentido es claro que el propósito fundamental del presente estudio se centra en establecer la capacidad predictiva de los exámenes de admisión de en la Facultad de Psicología, en relación con los resultados de los estudiantes en los tres diferentes cortes de notas, en los que se expresaría su aprendizaje.

Como se ha mencionado, además de los resultados de los dos exámenes practicados a los estudiantes, se encuentra la entrevista como elemento adicional en el proceso de admisión. Por ello, además del planteamiento anterior, también es importante establecer el porcentaje de varianza explicada con el estadístico R2 que, según Stalans (1995), es una medida de qué tan fuerte son las relaciones entre las variables criterio y la dependiente ya que otorga el porcentaje de varianza de la variable dependiente que es explicada por las variables criterio.

\section{Metodología}

Considerando que el interés general del estudio se centra en la posibilidad que tienen las pruebas de entrada de la Universidad y las pruebas del ICFES conocidas como Saber 11 de predecir los resultados del aprendizaje de los estudiantes, inferidos a partir de su rendimiento académico medido con las notas obtenidas en cada materia durante el primer semestre, el estudio es empírico, con metodología cuantitativa, ex post facto, retrospectivo, un grupo simple (León \& Montero, 2007), considerando que el conjunto de estudiantes seleccionados, tienen datos de las mismas variables.

Existen algunos otros estudios en el mismo sentido que el presente pero con la diferencia que utilizan procedimientos diferentes (correlaciones) y no establecen consideraciones particulares para la selección de la muestra, como es el hecho de seleccionar estudiantes que presentaron el "mismo" examen de estado, es decir, exámenes que tienen la misma estructura y cuyos resultados son comparables. Igualmente, mezclan diferentes cohortes de la institución en el programa sin considerar que las calificaciones que reciben los estudiantes en las asignaturas no son comparables entre una cohorte y otra por las múltiples variables que inciden al momento de emitir la calificación un docente, así sea el mismo docente y la misma asignatura con el mismo plan de estudios (Duque y Ortiz, 2013) (Montoya, D. 2011). 


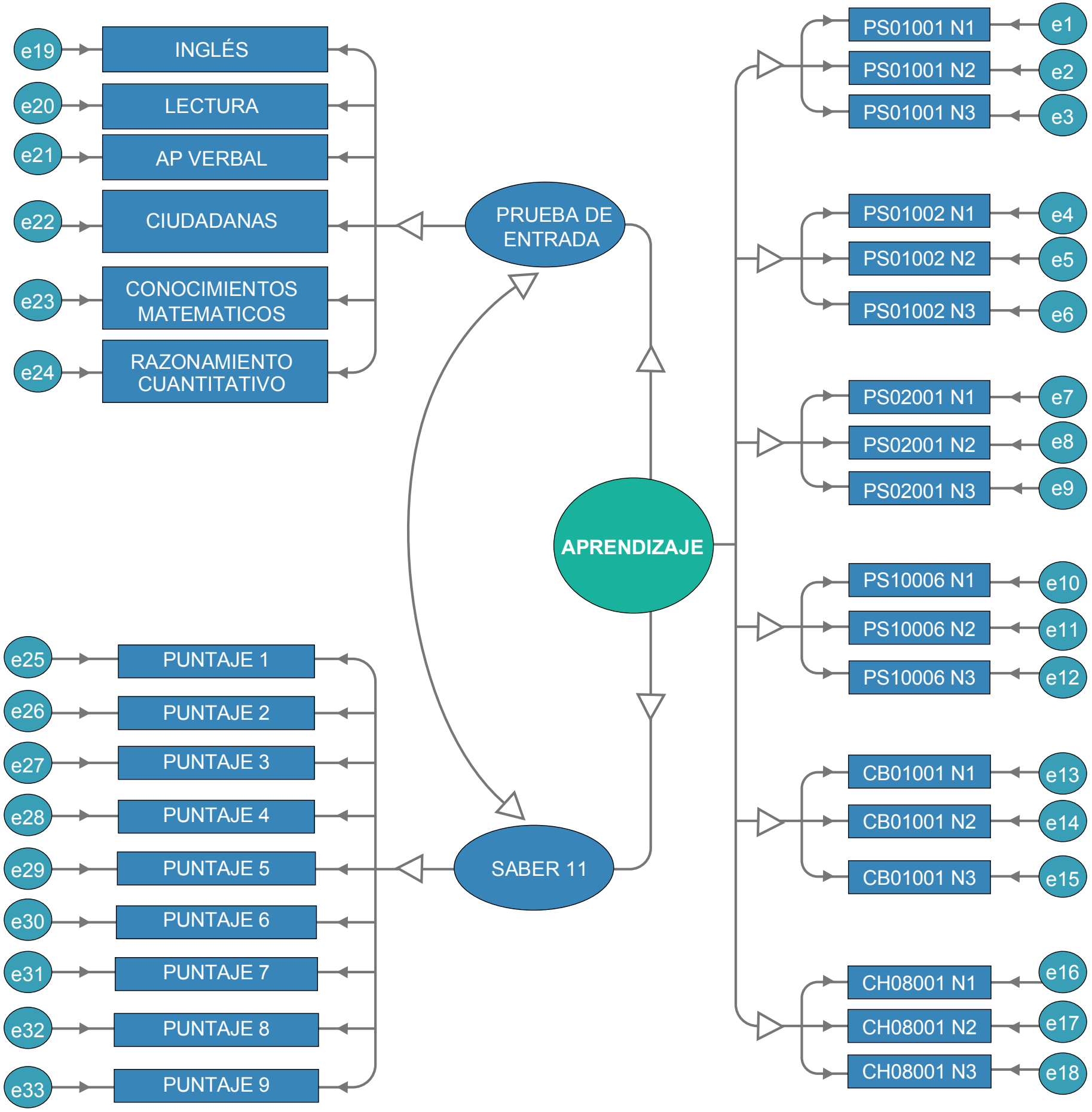

Figura 1. Modelo predictivo para el aprendizaje de los estudiantes de primer semestre de la carrera para el periodo I-2014.

Fuente: Autores. 
Los participantes en el estudio son todos los estudiantes que cursaron el primer semestre del programa de psicología durante el primer periodo académico de 2014. Este grupo de personas tienen unas características que los hacen idóneos para el estudio. Todos ingresaron al programa al mismo tiempo lo que implica que el efecto de la formación impartida es equitativo; todos presentaron la prueba de ingreso en el mismo lapso de tiempo y, adicionalmente, todos presentaron el mismo tipo de examen de estado o pruebas Saber 11. Con una característica adicional, tienen calificación en las mismas asignaturas del programa.

Para establecer la calidad de las relaciones entre las variables consideradas en el estudio se utilizó el método de Ecuaciones Estructurales con el programa AMOS, versión 16 (Arbuckle, 2011). Los modelos de Ecuaciones Estructurales contienen y utilizan una gran gama de procedimiento y técnicas estadísticas orientadas a probar y comprobar una serie de ecuaciones de regresión simultáneas (Yong-Yuan Chang, 2014). Las Ecuaciones Estructurales incluyen métodos como el path análisis, análisis factorial confirmatorio, regresiones lineales múltiples, entre otros (Arbuckle, 2011). El programa AMOS, Analysis of Moment Structures, realiza las Ecuaciones Estructurales de manera visual y sencilla por lo que se eligió para esta investigación.

\section{Resultados}

Para evitar la imputación de datos (procedimiento que se utiliza cuando falta algún dato en la matriz de información), se trabajó, solamente, con los estudiantes que tuvieran toda la información requerida para cada una de las variables del estudio. En total quedaron 177 estudiantes que cursaron primer semestre en el primer periodo académico del año 2014. No formaron parte del estudio quienes cancelaron asignaturas durante el semestre. El Modelo de Ecuaciones Estructurales utilizado en la presente investigación corresponde al que se observa en la figura 1 .

En la parte izquierda de la figura 1, se encuentran las variables predictoras (o variables criterio desde la perspectiva de Stalans, 1995) de la Prueba de Entrada y de las pruebas Saber 11, es decir los resultados de los 177 estudiantes en cada una de las sub pruebas de los dos exámenes. La relación se establece entre las tres variables no observadas (es decir que no tienen medida directa obtenida por las pruebas) Prueba de Entrada, Saber 11 y Aprendizaje. Esta última variable es medida con los resultados de los estudiantes en cada uno de los tres cortes de notas estipulados en la Universidad para cada una de las 6 asignaturas cursadas; es por esto que aparecen en grupos de a tres para cada asignatura.

Los resultados del estudio obtenidos con la metodología propuesta aparecen a continuación.

\begin{tabular}{|c|c|c|c|c|}
\hline Variable 1 & Relación & Variable 2 & Peso de regresión & $\mathrm{P}$ \\
\hline Aprendizaje & $<---$ & Prueba de entrada & 0.588 & $* * *$ \\
\hline Aprendizaje & $<---$ & Saber 11 & -0.308 & $* * *$ \\
\hline Inglés & $<---$ & Prueba de entrada & 0.569 & \\
\hline Lectura & $<---$ & Prueba de entrada & 0.52 & $* * *$ \\
\hline Aptitud verbal & $<---$ & Prueba de entrada & 0.637 & $* * *$ \\
\hline Puntaje 1 & $<---$ & Saber 11 & 0.454 & \\
\hline Puntaje 2 & $\begin{array}{l}<--- \\
\end{array}$ & Saber 11 & 0.195 & $* * *$ \\
\hline Puntaje 3 & $\begin{array}{ll}<--- \\
\end{array}$ & Saber 11 & 0.35 & $* * *$ \\
\hline Puntaje 4 & $\begin{array}{ll}<--- \\
\end{array}$ & Saber 11 & 0.446 & $* * *$ \\
\hline Puntaje 5 & $\begin{array}{ll}<--- \\
\end{array}$ & Saber 11 & 0.989 & $* * *$ \\
\hline Puntaje 6 & $\begin{array}{ll}<--- \\
\end{array}$ & Saber 11 & 0.133 & 0.088 \\
\hline Puntaje 7 & $<---$ & Saber 11 & 0.419 & $* * *$ \\
\hline Puntaje 8 & $<---$ & Saber 11 & 0.99 & $* * *$ \\
\hline Puntaje 9 & $<---$ & Saber 11 & 0.528 & $* * *$ \\
\hline Ps01001 n1 & $<---$ & Aprendizaje & 0.401 & \\
\hline Ps01001 n2 & $\begin{array}{ll}<--- \\
\end{array}$ & Aprendizaje & 0.464 & $* * *$ \\
\hline Ps01001 n3 & $\begin{array}{ll}<--- \\
\end{array}$ & Aprendizaje & 0.382 & $* * *$ \\
\hline Ch08001 n3 & $<---$ & Aprendizaje & 0.414 & $* * *$ \\
\hline Ch08001 n2 & $\begin{array}{l}<--- \\
\end{array}$ & Aprendizaje & 0.333 & $* * *$ \\
\hline Ch08001 n1 & $<---$ & Aprendizaje & 0.496 & $* * *$ \\
\hline Ps01002 n1 & $<---$ & Aprendizaje & 0.403 & $* * *$ \\
\hline Ps01002 n2 & $<---$ & Aprendizaje & 0.613 & $* * *$ \\
\hline Ps01002 n3 & $<---$ & Aprendizaje & 0.453 & $* * *$ \\
\hline Cb01001 n3 & $<---$ & Aprendizaje & 0.869 & $* * *$ \\
\hline $\mathrm{Cb} 01001 \mathrm{n} 2$ & $\begin{array}{ll}<--- \\
\end{array}$ & Aprendizaje & 0.807 & $* * *$ \\
\hline $\mathrm{Cb} 01001 \mathrm{n} 1$ & $\begin{array}{ll}<--- \\
\end{array}$ & Aprendizaje & 0.737 & $* * *$ \\
\hline Ps02001 n1 & <--- & Aprendizaje & 0.526 & $* * *$ \\
\hline Ps02001 n2 & $<---$ & Aprendizaje & 0.531 & $* * *$ \\
\hline Ps02001 n3 & $<<--$ & Aprendizaje & 0.441 & $* * *$ \\
\hline Ps10006 n1 & $<---$ & Aprendizaje & -0.007 & 0.931 \\
\hline Ps10006 n2 & $<---$ & Aprendizaje & 0.265 & $* * *$ \\
\hline Ps10006 n3 & <--- & Aprendizaje & 0.595 & $* * *$ \\
\hline $\begin{array}{l}\text { Razonamiento } \\
\text { cuantitativo }\end{array}$ & $<---$ & Prueba de entrada & 0.254 & $* * *$ \\
\hline $\begin{array}{l}\text { Conocimientos } \\
\text { matemáticos }\end{array}$ & $<---$ & Prueba de entrada & 0.38 & $* * *$ \\
\hline $\begin{array}{l}\text { Competencias } \\
\text { ciudadanas }\end{array}$ & $<---$ & Prueba de entrada & 0.349 & $* * *$ \\
\hline
\end{tabular}

Tabla 1. Peso de regresión y su significatividad estadística para las variables del estudio.

Fuente: Autores. 
El examen del Icfes -Saber 11-y la prueba de entrada como predictores del aprendizaje de los estudiantes de primer semestre de psicología de una universidad privada de Bogotá

Los asteriscos indican que los pesos de regresión son significativos estadísticamente. Se espera que el valor de $\mathrm{P}$ sea inferior a 0.05 para considerar que la relación es significativa. En el caso de este estudio la relación entre las variables PS1006 y aprendizaje y Puntaje 6 y Saber 11 son las únicas que no son significativas.

En términos del interés particular del estudio se comprueba que tanto la prueba de entrada como los resultados generales en Saber 11 predicen el aprendizaje de los estudiantes durante el primer semestre de estudio en el programa de Psicología. Como se observa en la tabla de datos, la prueba Saber 11 tiene una incidencia negativa en términos de la relación con las notas del estudiante y la prueba de entrada tiene una incidencia mayor y positiva.

El peso de regresión obtenido en la relación Prueba de Entrada y Aprendizaje (0.588) se basa en los datos estandarizados, por lo que su interpretación se realiza de la siguiente manera (Arbuckle, 2011): si en la prueba de entrada el resultado se aumenta en una desviación estándar, en los resultados de aprendizaje se aumenta 0.588 desviaciones estándar.

La correlación entre los resultados de Prueba de Entrada y Saber 11 es de 0.48 , que resulta significativo estadísticamente. Esto podría significar que no sería necesario utilizar uno de los dos exámenes como criterio de admisión, pero se requerirán estudios posteriores para definir este aspecto.

La varianza explicada (R2) por las variables de Prueba de Entrada y Saber 11 es del 27\% de la variable Aprendizaje, lo que implica que hay un $73 \%$ de varianza que no se explica por los resultados de estas pruebas. Este valor de la varianza que no es explicado por el modelo, requeriría de estudios posteriores que incluyan otras variables, incluso de corte personal. Sería interesante y quizás aumenta la explicación incluir la información que resulta de la entrevista de admisión y los resultados del cuestionario de personalidad que son aplicados a los aspirantes al programa. La inclusión de estas variables podría permitir determinar si en la predicción del aprendizaje de los estudiantes tienen un mayor peso variables de corte académico (competencias, habilidades y conocimientos) evaluadas por exámenes como prueba de entrada o Saber $11^{\circ}$, o variables de corte personal como la entrevista o escalas de personalidad.

\section{Discusión}

Es importante destacar que el planteamiento del propósito general del estudio se consigue. Es decir, se encuentra que hay una relación significativa entre las variables Prueba de Entrada y Saber 11 con el aprendizaje de los estudiantes. Al mismo tiempo, es muy importante considerar el hecho que los resultados de Saber 11 tiene una relación negativa de acuerdo con los resultados.

Este aspecto de la relación negativa es muy interesante de analizar ya que de acuerdo con la ley colombiana (ley 1324 del 13 de julio del 2009) las pruebas Saber 11 son un Examen de Estado que deben proporcionar información para el mejoramiento de la calidad de la educación $\mathrm{y}$, además, son requisito para ingresar a la Educación Superior. En este sentido de la medición de la calidad de la educación se entiende que proporcionan información para establecer planes de mejoramiento, lo que resulta un poco

\begin{tabular}{|c|c|}
\hline AÑO & NÚMERO ESTUDIANTES \\
\hline 2000 & 1 \\
\hline 2001 & 2 \\
\hline 2003 & 1 \\
\hline 2005 & 6 \\
\hline 2006 & 3 \\
\hline 2007 & 4 \\
\hline 2008 & 4 \\
\hline 2009 & 3 \\
\hline 2010 & 9 \\
\hline 2011 & 46 \\
\hline 2012 & 18 \\
\hline 2013 & 80 \\
\hline TOTAL & 177 \\
\hline
\end{tabular}

Tabla 2. Número de estudiantes de primer semestre según año de presentación de Saber 11.

Fuente: Autores. 
difícil si su relación con los resultados del aprendizaje es negativa. Podría pensarse que existen diferentes factores que pueden estar influyendo en que el resultado resulte en esa tendencia, como por ejemplo la fecha de presentación del examen Saber 11.

La gran mayoría de los estudiantes de primer semestre (más del 80\%) presentaron el examen después del 2010, así que los resultados son bastante actuales. No obstante, es un factor a considerar como interviniente que debe considerarse para profundizar un poco más en las posibilidades predictivas de la prueba Saber 11 .

Otro elemento consiste en la diversidad de las pruebas de este Examen. Como se sabe se evalúan áreas disciplinares tan disímiles como inglés, sociales, ciencias, matemáticas, entre otros. Es posible que la predicción del aprendizaje sea mucho mejor y con tendencia positiva para algunas áreas o pruebas y no para otras.

No se consideró pertinente realizar las relaciones de los datos con las subpruebas de Saber 11 debido a que no es clara la calidad de los instrumentos para producir resultados de este estilo, es decir, no se conocen datos de confiabilidad para esos resultados.

Esto llevaría a considerar que sería necesario hacer estudios por área y por institución hasta encontrar cuáles serían las pruebas o subpruebas apropiadas de este examen para tener en cuenta en el proceso de admisión de las Instituciones de Educación Superior (Rocha y Pardo, 1999). Pero esto, a su vez, llevaría a pensar que si se tiene una prueba institucional (como la Prueba de Entrada) que tiene buenas posibilidades de predicción, pues sobraría Saber 11, especialmente considerando que uno de los propósitos del examen, desde su creación (ley 80 de 1980) y de la versión del examen del año 2000 (Pardo, el al., 1999, p.12), plantea que el objetivo central de este examen conocido actualmente como Saber 11 es "servir como un criterio para el ingreso a la Educación Superior". Otro elemento muy importante del estudio es la relación significativa, en este caso positiva, entre la Prueba de Entrada de la Universidad y el resultado del aprendizaje de los estudiantes.
El peso de regresión observado es superior a 0.5 (el máximo es 1) lo que permite entender que puede resultar un buen criterio de predicción del desempeño. La mayoría de las pruebas se orientan a la medición de habilidades generales importantes para el desempeño en Educación Superior, lo que podría ser el soporte para los resultados encontrados. Tiene como ventaja, sobre la evaluación Saber 11 que todos los estudiantes analizados la presentaron al tiempo y en fecha muy cercana a la de ingreso a la universidad lo que aporta un elemento más a su favor en relación con las posibilidades de ser utilizada para evitar la deserción o el fracaso académico.

\section{Conclusiones}

Como conclusión general se tiene que tanto la prueba Saber 11 como la Prueba de Entrada se relacionan significativamente con los resultados de aprendizaje de los estudiantes en el primer semestre de la carrera, lo que las convierte en excelente instrumento para evitar el fracaso académico o la deserción por razones académicas, es decir las que tienen que ver con las posibilidades de aprendizaje de los estudiantes.

Para ello se requiere un uso adecuado, apropiado, de los resultados considerando las pruebas utilizadas en la medición, el tipo de resultados obtenidos y, con mayor énfasis, el tipo de relación encontrada para cada examen. Este último punto es muy importante puesto que malas interpretaciones (validez) pueden llevar a conclusiones erradas.

Adicionalmente, es importante profundizar mucho más en este tipo de resultados y realizar estudios que lleven a detectar, en detalle, las causas de estos resultados y establezcan las comparaciones necesarias entre grupos de estudiantes en variables como sexo, nivel socioeconómico, cultura educativa de la familia, entre otras, dando una mayor luz a lo que ocurre en Educación Superior.

Estos exámenes pueden ser útiles para evitar el fracaso escolar y la deserción por razones académicas, lo que contribuiría a resolver uno de los problemas centrales de la calidad de la educación en Colombia. 
El examen del Icfes -Saber 11-y la prueba de entrada como predictores del aprendizaje de los estudiantes de primer semestre de psicología de una universidad privada de Bogotá

\section{Referencias}

Arbuckle, J. (2011). IBM, SPSS, AMOS 20 User's Guide. Versión 20. AMOS Development Corporation.

Bernal-Velásquez, R. (Coordinador) (2013). Sistema nacional de evaluación estandarizada de la educación. Alineación del examen Saber 11. IcFEs: Bogotá.

Duque-Castillo, A. y Ortiz-Rodríguez, J. (2013). Pruebas ICFES Saber 11 y su relación con el desempeño académico en estudiantes de primer semestre de. Cuadernos Hispanoamericanos de psicología. Vol. 13 No. 1.

Forero, C., León, F \&Gutiérrez, G. (2014). Informe resultados prueba de entrada 2014-1. Programa de Psicología. Decanatura Académica, Universidad Católica de Colombia

Grupo de Evaluación de la Educación Básica y Media (1999). Antecedentes y marco legal del examen de Estado ICFES. Recuperado en http://186.113.12.12/discoext/ collections/0028/0001/02650001.pdf

León, O. y Montero, I. (2007). Métodos de Investigación en Psicología y Educación. McGraw-Hill Interamericana.

Ministerio de Educación Nacional (1980). Decreto 2343 "por el cual se reglamentan los exámenes de Estado para el Ingreso a la Educación Superior”. Diario Oficial 35603

Ministerio de Educación Nacional (1992). Ley 30 "por la cual se organiza el servicio público de la Educación Superior". http://www.mineducacion.gov.co/1621/ articles-85860_archivo_pdf.pdf

Ministerio de Educación Nacional (1998). Serie lineamientos Curriculares. http://www.mineducacion. gov.co/1621/w3-article-339975.html

Ministerio de Educación Nacional (2009). Ley 1324 “por la cual se fijan parámetros y criterios para organizar el sistema de evaluación de resultados de la calidad de la educación, se dictan normas para el fomento de una cultura de la evaluación, en procura de facilitar la inspección y vigilancia del Estado y se transforma el ICFEs".

Montoya, D. (2011). Estudio correlacional del examen Saber 11, el desempeño académico y los Exámenes de mitad de carrera-ECAMI- de los estudiantes de la Universidad del Rosario en el periodo 2007-2009. Universidad del Rosario. Bogotá.

Pardo, C., Rocha, A., Olaya, A., Sáenz, C., Jiménez, J., y Sánchez M. (1999). Nuevo examen de estado para el ingreso a la Educación Superior. Cambios para el siglo XXI. Propuesta general. IcFEs. Bogotá.

Pardo, C. (1999). Transformaciones en las pruebas para obtener resultados diferentes. ICFES. Bogotá.

Pedraza-Daza, F., Bernal- Velásquez, R \& Mora-Monje, A. (2014). Sistema nacional de evaluación estandarizada de la educación. Alineación del examen Saber 11. Lineamientos Generales 2014-2. ICFEs: Bogotá.

Stalans, L. (1995). Multidimensional Scaling. Capítulo 5. En: Reading and Understanding Multivariate Statistics. APA. Washington D.C.

Universidad Católica de Colombia (2014). Proceso de Inscripción y admisión. http://portalweb.ucatolica.edu.co/ easyWeb2/admisiones/pages.php/menu/993994094560/ id/4560/content/proceso-inscripcian-nuevos/

Yong-Yuan Chan (2014). Application of Structural Equation Modeling using AMOS. En: Academic Research. Recuperado en nov 27 de 2014, de: https:// www.google.com.co/webhp? sourceid=chromeinstant\&ion $=1 \&$ espv $=2 \&$ ie $=U T F-8 \# q=$ yong + Applicatio $\mathrm{n}+\mathrm{of}+$ Structural + Equation + Modeling + using + AMOS + in + Academic + Research 